\title{
Recherches sur la résistance des sols aux mala- dies. XV. Comparaison des populations de Pseudomonas fluorescents dans un sol résistant et un sol sensible aux fusarioses vasculaires
}

\author{
Philippe LEMANCEAU, Régine SAMSON (*), Claude ALABOUVETTE (**) \\ E.N.I.T.H., 2, rue Le Nôtre, F 49045 Angers Cedex \\ (*) I.N.R.A., Station de Pathologie végétale et de phytobactériologie, Centre de Recherches d'Angers, route de \\ St-Clément, Beaucouzé, F 49000 Angers \\ (**) I.N.R.A., Station de Recherches sur la Flore pathogène dans le sol, 17, rue Sully, F 21024 Dijon Cedex
}

Dans un article précédent, nous avons montré que la compétition pour le fer joue un rôle dans le mécanisme de résistance des sols de Chateaurenard. L'objet de cet article est donc d'apprécier la participation des Pseudomonas fluorescents à cette compétition.

Les populations de Pseudomonas fluorescents du sol résistant de Chateaurenard et du sol sensible de Carquefou sont comparées pour leur densité dans la terre adhérente (rhizoplan) ou non aux racines et pour leur aptitude à se développer en milieu carencé en fer. Les sols sont cultivés et maintenus à 3 valeurs différentes de potentiel hydrique : $\mathrm{pF} 1, \mathrm{pF} 2,5$ et $\mathrm{pF} 4$.

Au sein de la microflore aérobie totale, la fréquence de Pseudomonas fluorescents est 7 à 8 fois plus faible dans le sol résistant que dans le sol sensible. L'effectif des populations bactériennes, plus élevé dans le rhizoplan que dans la terre, varie peu avec les valeurs de potentiel hydrique auxquelles sont maintenus les sols. Les souches de Pseudomonas fluorescents collectées lors des dénombrements bactériens sont cultivées en milieu succinate additionné de concentrations croissantes de 8-hydroxyquinoléine. Chaque souche est caractérisée par la concentration minimale inhibitrice (CMI) de ce ligand qui diminue la disponibilité en fer du milieu. Les populations du rhizoplan et des sols à $\mathrm{pF} 1$ et $\mathrm{pF} 2,5$ sont respectivement mieux adaptées à la carence en fer que celles de la terre sans racine et des sols à $\mathrm{pF}$ 4. Les populations de Pseudomonas du sol résistant sont par ailleurs significativement plus sensibles à la carence en fer que celles du sol sensible.

La participation des Pseudomonas fluorescents à la compétition pour le fer et donc au mécanisme de résistance des sols de Chateaurenard semble réduite. Ils sont en effet moins nombreux en valeur relative et moins aptes à croître en milieu carencé en fer que ceux du sol sensible de Carquefou.

Mots clés additionnels : Fer, 8-hydroxyquinoléine, compétition nutritive, potentiel hydrique.

Studies on the disease suppressiveness of soils. XV. - Comparison of the fluorescent Pseudomonas population in a wilt-suppressive and a wilt-conducive soil.

In a previous paper, we showed that competition for iron plays a role in the mechanism of wilt suppressiveness of the soils from Chateaurenard. The aim of the present work was to test the role of fluorescent Pseudomonas in this competition. The bulk soil and rhizoplane populations of fluorescent Pseudomonas from a Chateaurenard suppressive soil and from a Carquefou conducive soil were compared in this respect and also for their ability to grow in a low iron-content medium. In addition, they were maintained at three different levels of hydric potential : $\mathrm{pF} 1, \mathrm{pF} 2.5$ and $\mathrm{pF}$ 4. The percentage of fluorescent Pseudomonas in the total aerobic microflora was 7 or 8 times lower in the suppressive soil than in the conducive soil. The size of the bacterial population, higher in the rhizoplane than in the bulk soil, depended on the soil matric potential. The strains of fluorescent Pseudomonas collected during bacterial counting were grown on a succinate medium containing increasing concentrations of 8-hydroxyquinoline. Each strain was characterized by its inhibitory minimal concentration (IMC) of this ligand, which decreases iron availability in the medium. The populations from the rhizoplane and from the soils maintained at $\mathrm{pF} 1$ and $\mathrm{pF} 2.5$ were better adapted to iron deficiency than those from the bulk soil and from the soils maintained at $\mathrm{pF} 4$. The populations of fluorescent Pseudomonas from the suppressive soil were significantly more sensitive to iron deficiency than those from the conducive soil. The participation of fluorescent Pseudomonas in competition for iron and also in the suppressiveness of the Chateaurenard soils seems limited, in so for as their relative level and ability to grow in an iron-deficient medium was lower than those from the conducive soil of Carquefou.

Additional key words : Iron, 8-hydroxyquinoline, competition for nutrients, matric potential. 


\section{INTRODUCTION}

Dans un article précédent (LEMANCEAU et al., 1987) nous avons démontré que la compétition pour le fer est un des phénomènes qui conditionnent le niveau de réceptivité des sols aux fusarioses vasculaires. En effet, l'apport d'un ligand (EDDHA) à forte affinité pour le fer diminue la gravité de la maladie alors que l'apport de fer chélaté à EDTA augmente la gravité de la fusariose et ceci aussi bien dans le sol résistant de Chateaurenard que dans le sol sensible de Carquefou. Ces résultats sont comparables à ceux obtenus dans les sols de Salinas Valley par SCHER \& BAKER (1982) qui attribuent aux populations de Pseudomonas fluorescents l'intense compétition pour le fer, responsable de la résistance de ces sols. Dans des conditions de $\mathrm{pH}$ élevé et de faible concentration en fer facilement extractible, les Pseudomonas fluorescents produisent des sidérophores, les pyoverdines, qui forment avec le fer ferrique ( $\mathrm{Fe}$ III) des complexes stables (MEYER \& ABDAllaH, 1978). L'affinité pour l'ion Fe III des pyoverdines étant supérieure à celle des fusarinines, sidérophores produits par les Fusarium (EMERY, 1965) ces derniers sont incapables de se procurer dans le milieu le fer indispensable à la germination des chlamydospores et à l'allongement des tubes germinatifs (SNEH et al., 1984 ; ELAD \& BAKER, 1985). SCHER \& BAKER $(1980,1982)$ ont ainsi pu augmenter le niveau de résistance de certains sols, soit par apport de chélates de synthèse, soit par introduction de Pseudomonas fluorescents.

Après avoir démontré précédemment en utilisant des chélates de synthèse que la disponibilité en fer conditionne le niveau de réceptivité aux fusarioses du sol sensible de Carquefou et du sol résistant de Chateaurenard, il est indispensable d'analyser les populations de Pseudomonas de ces deux sols. Les populations sont comparées à la fois sur le plan quantitatif et sur le plan qualitatif. Il est en effet démontré que l'intensité de la synthèse des sidérophores varie selon les souches de Pseudomonas fluorescents étudiées (LEMANCEAU \& SAMSON, 1983) et que l'affinité pour le fer des sidérophores dépend de leur structure chimique (TEINTZE et al., 1981 ; DEMANGE et al., 1986 ; MEYER et al., 1987). Il est donc utile de caractériser l'activité des populations de Pseudomonas fluorescents des sols résistant et sensible pour évaluer leur contribution au phénomène de compétition pour le fer et donc aux mécanismes de réceptivité des sols aux fusarioses vasculaires.

\section{MATÉRIEL ET MÉTHODES}

\section{A. Sols et conditions expérimentales}

Les caractéristiques des sols résistant de Chateaurenard et sensible de Carquefou ont été présentées précédemment (LEMANCEAU et al., 1988b). Le potentiel hydrique des sols étant un facteur écologique susceptible de modifier l'équilibre des populations bactériennes, toutes les expérimentations ont été réalisées dans des conditions d'humidité contrôlée. La figure 1 indique pour chacun des deux sols, la teneur pondérale en eau, exprimée en $\mathrm{g}$ d'eau par $\mathrm{g}$ de sol, correspondant

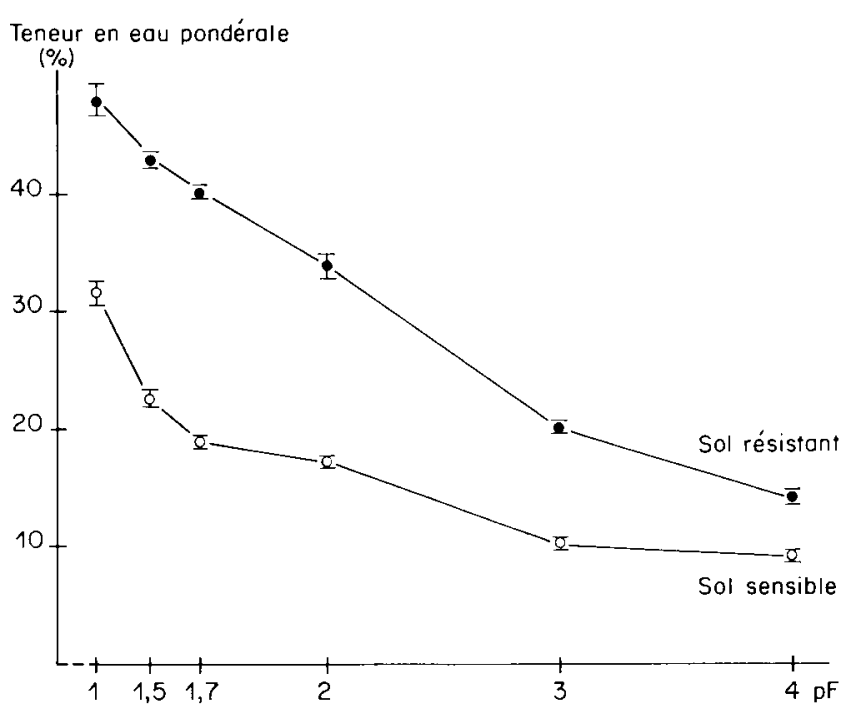

Figure 1

Potentiel hydrique et teneur en eau du sol résistant de Chateaurenard et du sol sensible de Carquefou. Moyennes de 3 répétitions suivies de leurs écarts types (I).

Matric potential and water content of the suppressive soil from Chateaurenard and of the conducive soil from Carquefou. Means of 3 replicates followed by their standard deviations (I).

à différentes valeurs de potentiel hydrique imposées par dépression entre $\mathrm{pF} 1$ et $\mathrm{pF} 2$ et par pression de $\mathrm{pF} 2,5$ à $\mathrm{pF} 4$.

Les sols sont répartis à raison de $500 \mathrm{ml}$ par pot, 10 graines de lin, variété "Hera », sont semées et l'humidité est ajustée par pesée à la valeur correspondant à $\mathrm{pF}$ 2,5. Après l'émergence des plantules, l'humidité des sols est amenée aux potentiels $\mathrm{pF} 1$, $\mathrm{pF} 2,5$ et $\mathrm{pF} 4$ selon les traitements, puis maintenue à ces valeurs par pesée journalière. La culture est réalisée pendant 4 semaines, en chambre climatisée, à une température de $25^{\circ} \mathrm{C}$ le jour, $20^{\circ} \mathrm{C}$ la nuit, et à une photopériode de $16 \mathrm{~h}$.

\section{B. Analyse des populations bactériennes}

Les plantes étant arrachées, on analyse séparément un échantillon de terre sans racine et un échantillon de terre adhérente aux racines (rhizoplan). Les échantillons de terre ou de racines grossièrement nettoyées sont agités pendant $1 \mathrm{~h}$ dans une solution dispersante (Olivier \& Guillaumes, 1976). Les dilutions successives de 10 en 10 sont effectuées dans l'eau stérile. La dessiccation à $105^{\circ} \mathrm{C}$ de la suspension mère permet ensuite d'exprimer les résultats par le nombre de c.f.u. (" colony forming unit») par $\mathrm{g}$ de terre sèche. Les étalements sont réalisés sur le milieu B de King (KING et al., 1954) pour le dénombrement de la flore bactérienne aérobie "totale », sur le milieu B de King additionné de cristal violet $(2 \mathrm{mg} / \mathrm{l})$ pour le dénombrement des bactéries à réaction de Gram négative. Les colonies de Pseudomonas fluorescents sont dénombrées sous lumière ultra-violette $(\lambda=366 \mathrm{~nm})$. Pour chaque traitement expérimental, on réalise 3 séries de suspension-dilution. Pour chacune d'elles, les étalements sont réalisés sur 4 boîtes de Petri, pour chaque milieu. La lecture est effectuée après $4 \mathrm{j}$ d'incubation à $25^{\circ} \mathrm{C}$. 
Afin d'apprécier qualitativement l'aptitude des populations de Pseudomonas à se développer en milieu carencé en fer, une collection comprenant respectivement 51 et 56 souches pour les sols résistant et sensible est constituée par isolement et purification de 8 à 10 colonies par traitement expérimental. Les différentes espèces et biovars de Pseudomonas sont déterminés selon les critères de PALLERONI (1984). L'aptitude des souches à se développer en milieu carencé en fer est déterminée par culture sur milieu succinate (MEYER \& ABDALLAH, 1978) additionné de concentrations croissantes de 8-hydroxyquinoléine (2 à $128 \mathrm{ppm}$ ) ligand organique à forte affinité pour le fer (SCHIPPERS et al., 1986 ; LEMANCEAU et al., 1987). Les cultures sont réalisées en plaques de microtitration, 4 cupules sont ensemencées pour chaque concentration de ligand, la croissance bactérienne est mesurée par turbidité $(\lambda=650 \mathrm{~nm})$ au lecteur automatique, $4 \mathrm{j}$ après l'ensemencement. On détermine ainsi la concentration minimum de 8-hydroxyquinoléine qui inhibe la croissance des Pseudomonas (CMI). Les distributions des populations de Pseudomonas dans les différentes classes de CMI sont comparées par le test statistique 2 i (SOKAL \& ROHLF, 1969).

\section{RÉSULTATS}

\section{A. Niveau des populations bactériennes}

La figure 2 présente l'ensemble des résultats relatifs aux densités des populations bactériennes totales, à réaction de Gram négative et Pseudomonas fluorescents déterminées dans le rhizoplan et la terre des sols résistant et sensible maintenues pendant 4 semaines à 3 valeurs différentes de potentiel hydrique. En ce qui concerne la densité de la population bactérienne totale il n'apparaît pas de différence marquée ni en fonction des sols, ni en fonction des valeurs de potentiel hydrique. Cependant, le niveau des populations bactériennes est toujours légèrement supérieur dans la terre adhérente aux racines. Les populations à réaction de Gram négative présentent les mêmes tendances que les populations totales. La densité des Pseudomonas fluorescents ne semble pas différente selon le potentiel hydrique des sols, mais elle est toujours plus importante dans la terre du rhizoplan. De plus, les Pseudomonas fluorescents semblent plus fréquents dans le sol sensible de Carquefou que dans le sol résistant de Chateaurenard; leur densité par rapport à la flore

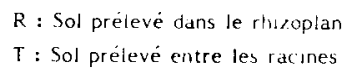

SOL RESISTANT (CHATEAURENARD)
Population "totale"

Q79 Bactéries à réaction de Gram négative

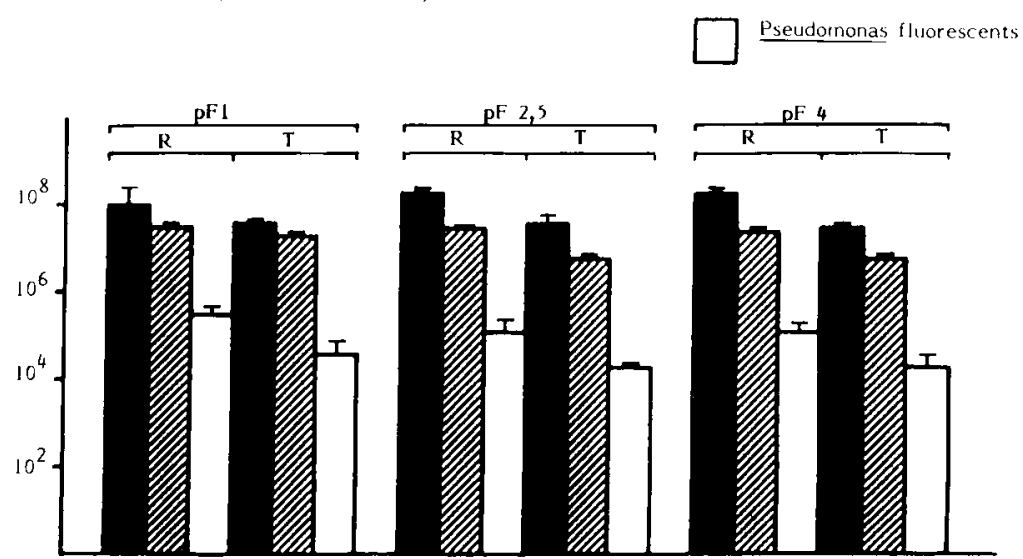

SOL SENSIBLE (CARQUEFOU)

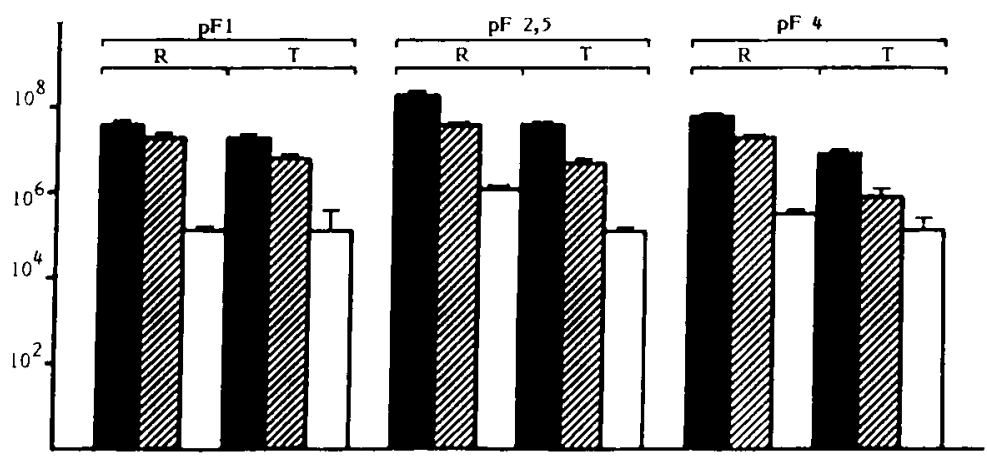

Densité des populations bactériennes du sol résistant (Chateaurenard) et $d u$ sol sensible (Carquefou) maintenus à différentes valeurs de potentiel hydrique. (Même légende que figure 1).
Density of the bacterial populations of the suppressive soil (Chateaurenard) and of the conducive soil (Carquefou) maintained at different levels of matric potential. (Same legend as figure 1). 
bactérienne totale y est en moyenne 7 à 8 fois supérieure.

\section{B. Caractéristiques des populations de Pseudomonas}

Les Pseudomonas fluorescents appartiennent aux espèces $P$. putida et $P$. fluorescents, cette espèce étant représentée par les biovars 2,3 et surtout par le biovar 5 (tabl. 1). L'espèce $P$. putida est mieux représentée dans le sol sensible que dans le sol résistant.

TABLEAU 1

Composition des populations de Pseudomonas fluorescents du sol résistant de Chateaurenard et du sol sensible de Carquefou. Composition of the populations of fluorescent Pseudomonas from the suppressive soil of Chateaurenard and from the conducive soil of Carquefou.

\begin{tabular}{lcccccc}
\hline \hline \multirow{2}{*}{ Sols } & \multicolumn{4}{c}{ Pseudomonas fluorescens } & $\begin{array}{c}\text { Pseudo- } \\
\text { monas }\end{array}$ & Total \\
\cline { 2 - 7 } & Biovar 2 Biovar & 3 Biovar 5 & putida & \\
\hline $\begin{array}{l}\text { Résistant } \\
\text { (Chateaurenard) }\end{array}$ & 10 & 35 & 29 & 26 & 100 \\
\hline $\begin{array}{l}\text { Sensible } \\
\text { (Carquefou) }\end{array}$ & - & 6 & 53 & 42 & 100 \\
\hline \hline
\end{tabular}

La figure 3 indique pour chacun des sols, la répartition des souches de Pseudomonas dans chaque classe de concentration minimale inhibitrice (CMI) de 8hydroxyquinoléine. Les populations de Pseudomonas isolées du sol résistant et du sol sensible présentent une distribution significativement différente au seuil de $0,1 \mathrm{p} .100$. Les classes qui possèdent les effectifs les plus importants sont respectivement 8 et $32 \mathrm{ppm}$ de 8-hydroxyquinoléine pour le sol résistant et le sol sensible. De plus, dans ce sol sensible, il existe des souches capables de se développer à la concentration de $128 \mathrm{ppm}$ alors qu'en sol résistant aucune souche ne se développe au-delà de $32 \mathrm{ppm}$.

La figure 4 présente la distribution dans les différentes classes de CMI des populations de Pseudomonas isolées du rhizoplan du lin ou de la terre dépourvue de racine des sols résistant et sensible. Selon les sols, la plus grande sensibilité des populations du rhizoplan à la 8-hydroxyquinoléine est significative (sol de Chateaurenard, seuil 5 p. 100) ou non (sol de Carquefou).

La figure 5 montre l'influence du potentiel hydrique sur la distribution des populations de Pseudomonas en fonction de leur aptitude à se développer en milieu carencé en fer. Les différences enregistrées entre $\mathrm{pF} 1$ et $\mathrm{pF} 2,5$ sont peu (sol résistant) ou pas (sol sensible) significatives. Par contre, quel que soit le sol considéré, les populations isolées des sols maintenus à $\mathrm{pF} 4$ sont très significativement (seuil 0,1 p. 100) plus sensibles à la 8-hydroxyquinoléine que celles des sols cultivés à $\mathrm{pF} 1$ ou à $\mathrm{pF} 2,5$. Ainsi pour le sol de Chateaurenard, les classes qui présentent l'effectif le plus important sont $8 \mathrm{ppm}$ à $\mathrm{pF} 1$ et $\mathrm{pF} 2,5$ et $4 \mathrm{ppm}$ à $\mathrm{pF} 4$. Cette différence est encore plus marquée dans le sol de Carquefou : $32 \mathrm{ppm}$ à pF 1 et pF 2,5 contre 8 ppm à pF 4.
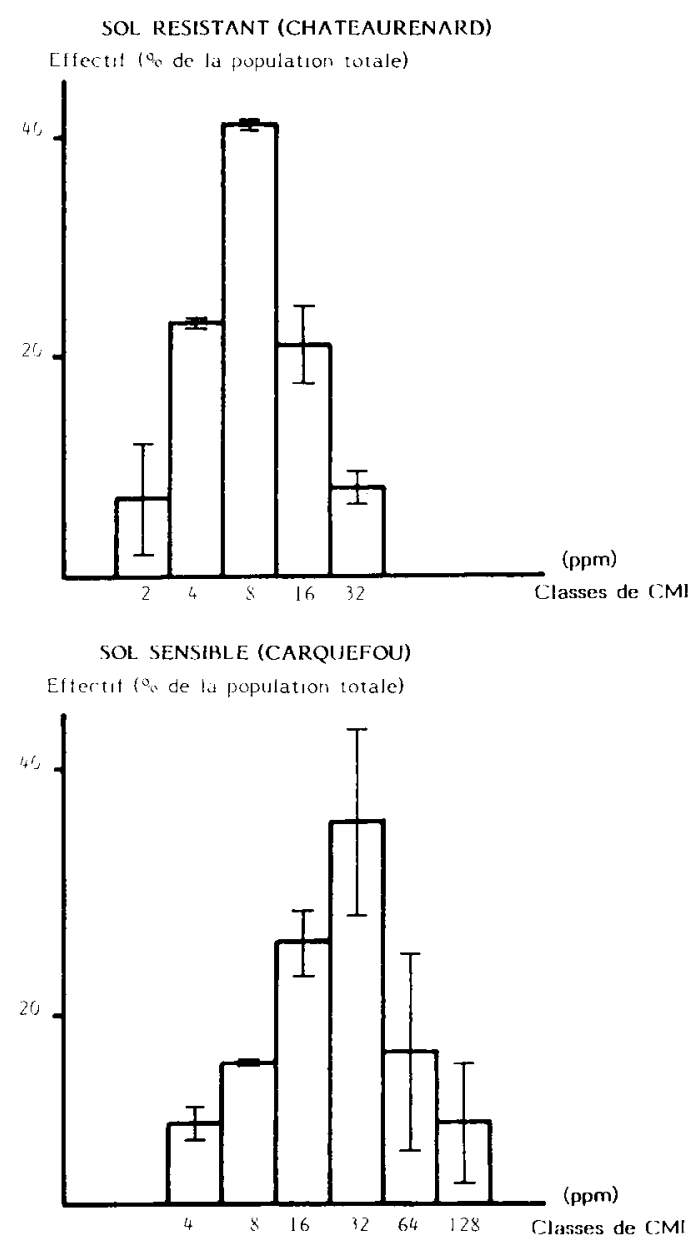

Figure 3

Distribution des populations de Pseudomonas fluorescents du sol résistant (Chateaurenard) et du sol sensible (Carquefou) selon leur sensibilité à la 8-hydroxyquinoléine. (Même légende que figure I).

Distribution of the fluorescent Pseudomonas populations from the suppressive soil (Chateaurenard) and from the conducive soil (Carquefou) depending on their sensitivity to 8-hydroxyquinoline. (Same legend as figure 1).

\section{DISCUSSION}

L'objectif de ce travail était de comparer les populations bactériennes du sol résistant de Chateaurenard et du sol sensible de Carquefou. Les résultats acquis indiquent que les densités des populations bactériennes totales et à Gram négatif sont analogues. Les techniques de suspension-dilution utilisées sont peu précises (LOPER et al., 1984) et ne permettent pas de révéler tous les microorganismes du sol. Elles autorisent cependant les comparaisons entre traitements expérimentaux. Les résultats obtenus confirment d'ailleurs une observation classique (DOMMERGUES \& MANGENOT, 1970) à savoir que les populations bactériennes sont plus abondantes dans la terre adhérente aux racines que dans la terre éloignée de la surface racinaire. Ces résultats démontrent par ailleurs que les Pseudomonas fluorescents sont plus abondants dans le sol sensible de Carquefou que dans le sol résistant de Chateaurenard. Ces indications sont difficiles à comparer à celles d'autres auteurs car les études prenant en considération l'ensemble de la population de Pseudomonas fluorescents sont rares (SANDS \& ROVIRA, 1971 ; SAMSON et al., 1987 ; XU \& CROSS, 
SOL RESISTANT (CHATEAURENARD)

RHIZOPLAN
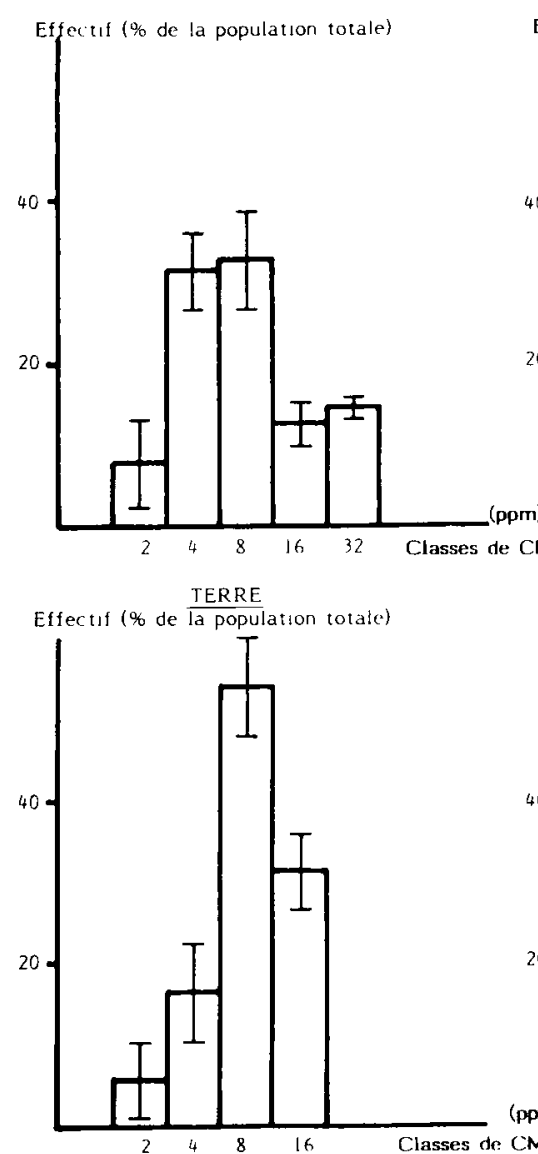

SOL SENSIBLE (CARQUEFOU) RHIZOPLAN
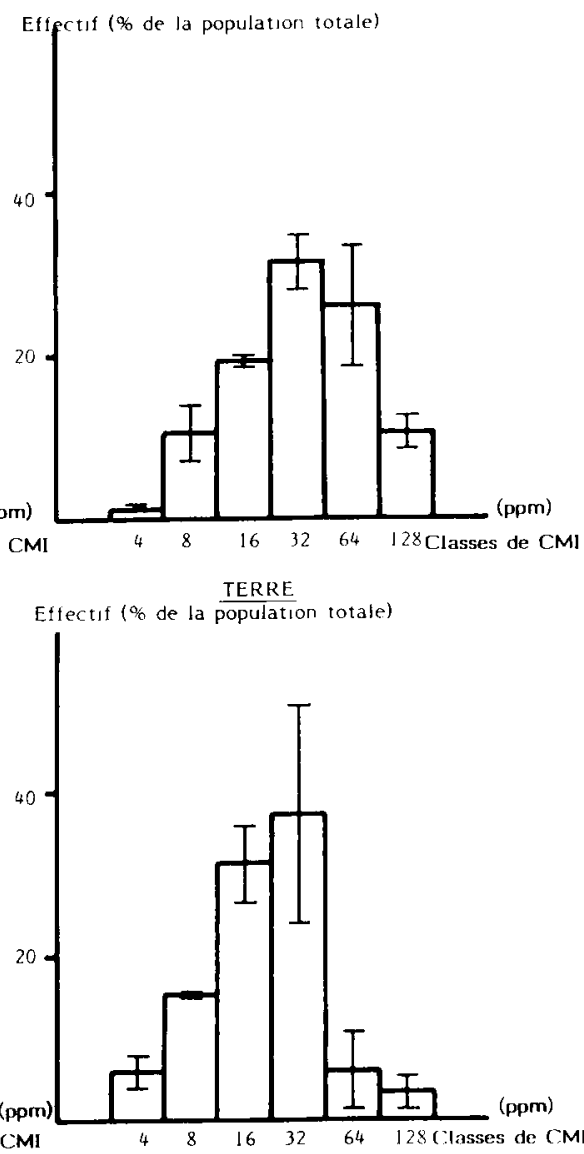

Figure 4

Distribution des populations de Pseudomonas fluorescents du rhizoplan et de la terre selon leur sensibilité à la 8 hydroxyquinoléine. (Même légende que figure 1).
Distribution of the fluorescent Pseudomonas populations from the rhizoplane and from the bulk soil depending on their sensivity to 8hydroxyquinoline. (Same legend as figure I).
1986). Généralement, les travaux consacrés aux mécanismes de résistance des sols aux maladies considèrent la dynamique d'une souche particulière de Pseudomonas au niveau du sol ou de la rhizosphère (SCHER et al., 1984 ; DUPLER \& BAKER, 1984).

De nombreux travaux insistent sur le rôle déterminant de l'humidité et de l'aération du sol sur le développement de la microflore tellurique (GRIFFIN, 1969 ; COOK \& BAKER, 1983). En ce qui concerne les Pseudomonas, DUPLER \& BAKER (1984) montrent que la colonisation du sol est, pour une souche déterminée, plus importante pour de fortes humidités du sol et devient nulle au-delà de $\mathrm{pF} 2$. Nos résultats n'indiquent aucune influence du potentiel hydrique sur la densité des populations bactériennes analysées. Cette stabilité apparente peut s'expliquer par la diversité des populations naturelles étudiées qui occulte les variations individuelles de certaines souches. D'ailleurs HowIE et al. (1987) précisent que la sensibilité aux variations du potentiel hydrique d'une souche introduite est supérieure à celles des populations autochtones. De plus, il faut remarquer que le potentiel hydrique des sols est un facteur difficile à maintenir à une valeur déterminée, des variations importantes peuvent être enregistrées selon la méthodologie utilisée.
La comparaison quantitative des populations de Pseudomonas a été complétée par la détermination de leurs aptitudes respectives à se développer en milieu carencé en fer. Cette aptitude a été appréciée par l'emploi de concentrations croissantes de 8hydroxyquinoléine, ligand ayant une forte affinité pour le fer. Dès 1936, DuGAY signalait l'action bactériostatique du sulfate double d'orthoxyquinoléine et de potassium. Mais la compréhension du mode d'action est plus récente (SoUSSY et al., 1977). Il repose sur le pouvoir chélateur de cette molécule qui crée, dans le milieu, une carence en oligo-éléments indispensables à la croissance des microorganismes. L'intérêt d'employer la 8-hydroxyquinoléine pour sélectionner des Pseudomonas fluorescents stimulant la croissance des plantes a été démontrée par SCHIPPERS et al. (1986). Nous avons vérifié que l'inhibition de la croissance des souches de Pseudomonas en présence de 8-hydroxyquinoléine est effectivement due à la carence en fer. L'inhibition de croissance de 5 souches présentant des CMI de 4 à 64 ppm ne se manifeste pas en présence d'une forte concentration (128 ppm) du chélate Fer-8-hydroxyquinoléine. La détermination de la concentration minimum inhibitrice de 8-hydroxyquinoléine est donc bien un moyen 

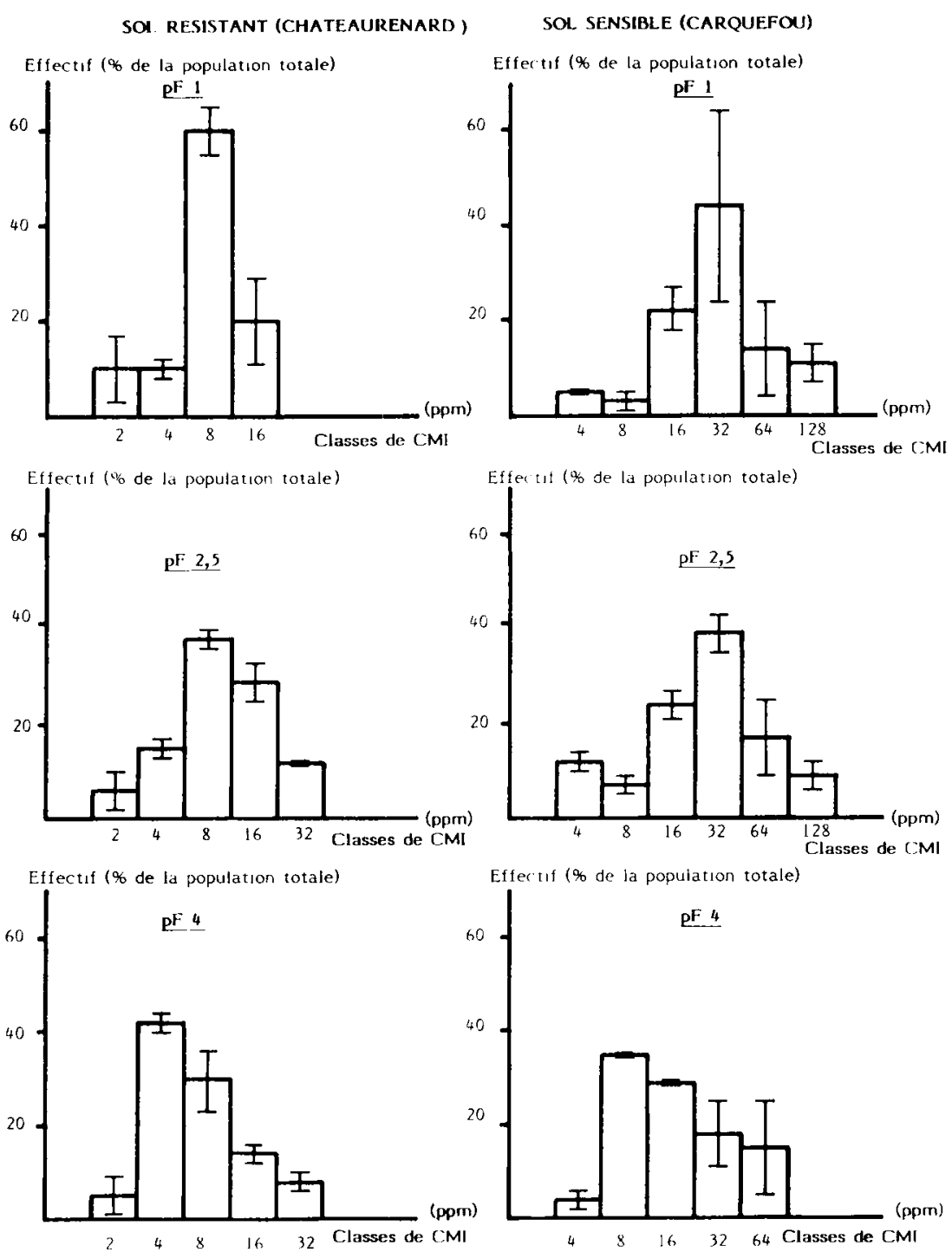

Figure 5

Distribution des populations de Pseudomonas fluorescents des sols maintenus à $p F 1, p F 2,5, p F 4$ selon leur sensibilité à la 8hydroxyquinoléine. (Même légende que figure 1).

d'apprécier l'aptitude des souches de Pseudomonas à se développer en milieu plus ou moins carencé en fer.

Les résultats obtenus s'interprètent d'ailleurs logiquement par rapport à la disponibilité en fer des sols. Plusieurs auteurs indiquent que la compétition pour le fer est plus intense dans la rhizosphère que dans le sol lui-même (SNEH et al., 1984 ; BAKER et al., 1986). Les résultats présentés figure 3 indiquent en effet que les populations de Pseudomonas isolées de la terre associée aux racines sont moins sensibles que celles du sol à l'inhibition induite par la 8-hydroxyquinoléine. Elles sont donc mieux adaptées à la compétition pour le fer.

L'aération du sol, qui détermine le potentiel redox, influence l'équilibre fer ferreux/fer ferrique (LINDSAY, 1974). A pF 4, la porosité est essentiellement occupée par l'air ; la disponibilité en fer ferrique est plus importante qu'à $\mathrm{pF} 1$. Les Pseudomonas isolés du sol maintenu à $\mathrm{pF} 4$ sont effectivement plus sensibles à la 8-hydroxyquinoléine que ceux isolés du sol maintenu à $\mathrm{pF} 1$; ils sont donc moins aptes à la compétition pour le fer.

Distribution of the fluorescent Pseudomonas populations from the soils maintained at $p F 1, p F 2.5, p F 4$ depending on their sensivity to the 8-hydroxyquinoline. (Same legend as figure 1).

Ces diverses observations indiquent que ces résultats de laboratoire ont une réelle signification écologique. Dans ce cas, il est surprenant de constater que les populations de Pseudomonas isolées du sol résistant de Chateaurenard sont globalement moins aptes à se développer en milieu carencé en fer que les populations isolées du sol sensible de Carquefou. Cette différence est manifeste quelles que soient les conditions expérimentales ( $\mathrm{pF} 1, \mathrm{pF} 2,5$ et $\mathrm{pF} 4)$. Or, il a été démontré que la concentration en fer facilement extractible est, dans le sol de Chateaurenard, 15 fois plus faible que dans le sol de Carquefou (LEMANCEAU et al., 1988). Il ne semble donc pas exister de corrélation entre la faible disponibilité en fer des sols de Chateaurenard et l'aptitude des souches de Pseudomonas à se développer en milieu carencé. La résistance de ces sols ne serait donc pas due à l'activité chélatrice du fer des Pseudomonas fluorescents.

Ces résultats sont à rapprocher de ceux de LOCKWOOD \& SCHIPPERS (1984) montrant que dans les sols ayant un niveau de fongistase élevé, le premier facteur de sélection des microorganismes est la compétition 
pour l'énergie et non pas la compétition pour le fer. Les sols de Chateaurenard possèdent un niveau de fongistase élevé et la compétition pour le carbone y joue un rôle déterminant (ALABOUVETTE et al., 1985).

On peut donc penser que la compétition pour le fer, comme celle pour le carbone, est liée à l'abondance et à l'activité de la biomasse microbienne.

\section{REMERCIEMENTS}

Nous remercions L. M. RIVIÈre (ENITH - ANGERS) pour l'aide qu'il nous a apportée lors des mesures de potentiel hydrique et pour les nombreux conseils dont il nous a fait profiter.

Reçu le 5 mai 1987. Accepté le 3 décembre 1987.

\section{RÉFÉRENCES BIBLIOGRAPHIQUES}

Alabouvette C., Couteaudier Y., Louvet J., 1985. Recherches sur la résistance des sols aux maladies. XII. Activité respiratoire dans un sol résistant et un sol sensible aux fusarioses vasculaires enrichis en glucose. Agronomie, 5 (1), 69-72.

Baker R., Elad Y., Sneh B., 1986. Physical, biological and host factors in iron competition in soils. 77-84. In : T. R. Swinburne "Iron, siderophores, and plant diseases". NATO Adv. Res. Workshop, U.K., Plenum Press New York and London.

Cook R. J., Baker K. F., 1983. The nature and practice of biological control of plant pathogens. Am. Phytopathol. Soc., St Paul MN, USA, $539 \mathrm{p}$.

Demange P., Wendenbaum S., Bateman A., Dell A., Meyer J. M., Abdallah M. A., 1986. Bacterial siderophores of pyoverdines and related compounds. 131-147. In : T. R. Swinburne "Iron, siderophores, and plant diseases". NATO Adv. Res. Workshop, U.K., Plenum Press New York and London.

Dommergues Y., Mangenot F., 1970. Ecologie microbienne du sol. Masson et Cie, Editeurs, 796 p., 180 figures, 101 tableaux.

Dugay M., 1936. Le sulfate double d'orthoxyquinoléine et de potassium. Thèse Doc. Médecine, Fac. Médecine, Paris, $136 \mathrm{p}$.

Dupler M., Baker R., 1984. Survival of Pseudomonas putida, a biological control agent, in soil. Phytopathology, 74, 195-200.

Elad Y., Baker R., 1985. The role of competition for iron and carbon in suppression of chlamydospore germination of Fusarium spp. by Pseudomonas spp., Phytopathology, 75, 1053-1059.

Emery T., 1965. Isolation, characterization, and properties of fusarinine a hydroxamic acid derivative of ornithine. Biochemistry, 4, $1410-1417$.

Griffin D. M., 1969. Soil water in the ecology of fungi. Ann. Rev. Phytopathol, 7, 289-310.

Howie W. J., Cook R. J., Weller D. M., 1987. The effect of soil matric potential and cell motility on wheat root colonization by fluorescent pseudomonas suppressive to take-all. Phytopathology, 77, 286-292.

King E. O., Ward M. K., Raney D. E., 1954. Two simple media for the demonstration of pyocanin and fluorescin. J. Lab. Clin. Med., 44, 301-307.

Lemanceau P., Samson R., 1983. Relation entre quelques caractéristiques in vitro de 10 Pseudomonas fluorescents et leur effet sur la croissance du haricot (Phaseolus vulgaris), 327-328. In : "Les antagonismes microbiens». $24^{\mathrm{e}}$ colloque S.F.P. Coll. I.N.R.A. ${ }^{\circ}$ 18. Ed. I.N.R.A. Publ., Versailles.

Lemanceau P., Samson R., Rivière L. M., Alabouvette C., 1987. Effet de la 8-hydroxyquinoléine sur la croissance, in vitro, de populations telluriques de Pseudomonas fluorescents, 244-245. In : J. M. Legay «Biologie des populations ». Coll. Nat. CNRS. IASBE, Univ. Claude Bernard, Lyon I.

Lemanceau P., Alabouvette C., Couteaudier Y., 1988. Recherches sur la résistance des sols aux maladies. XIV. Modification du niveau de réceptivité d'un sol résistant et d'un sol sensible aux fusarioses vasculaires en réponse à des apports de fer et de glucose. Agronomie, 8 (2), 155-162.

Lindsay W. L., 1974. Role of chelation in micronutrient avaibilir! 507-527. In : E. W. Corson "The plant root and its environment". University Press Virginia, Charlottesville, $691 \mathrm{p}$.

Lockwood J. L., Schippers B., 1984. Evaluation of siderophores as a factor in soil mycostasis. Trans. Br. Mycol. Soc., 82 (4), 589-594.
Loper J. E., Suslow T. V., Shroth M. N., 1984. Lognormal distribution of bacterial populations in the rhizosphere. Phytopathology, 74, 1454-1460.

Meyer J. M., Abdallah M. A., 1978. The fluorescent pigment of Pseudomonas fluorescens: biosynthesis, purification and physicochemical properties. J. gen. Microbiol., 107, 319-328.

Meyer J. M., Halle F., Hohnadel O., Lemanceau P., Ratefiarivelo H., 1987. Siderophores of Pseudomonas. Biological properties, 189205. In : D. Van Der Helm, J. Neilands, G. Winkelmann "Iron transport in microbes plants and animals". $\mathrm{VCH}$ Weinheim (sous presse).

Olivier J. M., Guillaumes J., 1976. Etude écologique des composts de champignonnière. Ann. Phytopathol., 8 (3), 283-301.

Palleroni N. J., 1984. Pseudomonas, 141-199. In : R. E. Buchanan, N. E. Gibbons 'Bergey's Manual of systematic bacteriology”. Vol. 1, Williams, Wilkinseds, eds Baltimore.

Samson R., Lemanceau P., Perrin R., Alabouvette C., 1987. Diversité des populations de Pseudomonas fluorescens et putida dans différents sols cultivés, 269. In : J. M. Legay "Biologie des populations ». Coll. Nat. CNRS. IASBE, Univ. Claude Bernard, Lyon I.

Sands D. C., Rovira A. D., 1971. Pseudomonas fluorescens. Biotype G., the dominant fluorescent pseudomonad in South Australia soili, and wheat rhizospheres. J. appl. Bacteriol., 34, 261-275.

Scher F. M., Baker R., 1980. Mechanism of biological control in a Fusarium-suppressive soil. Phytopathology, 70, 412-417.

Scher F. M., Baker R., 1982. Effect of Pseudomonas putida and a synthetic iron chelator on induction of soil suppressiveness to Fusarium wilt pathogens. Phytopathology, 72, 1567-1573.

Scher F. M., Dupler M., Baker R., 1984. Effect of a synthetic iron chelate on population densities of Fusarium oxysporum and the biological agent Pseudomonas putida in soil. Can. J. Microbiol., 30, 1271-1275.

Schippers B., Geels F. P., Baker P. A. H. M., Baker A. W., Weisbeek P. J., Lugtengerg B., 1986. Methods of studying plant growth stimulating pseudomonads: problems and progress, 149-154. In: T. R. Swinburne "Iron, siderophores, and plant diseases". NATO Adv. Res. Workshop, U.K., Plenum Press New York and London.

Sneh B., Dupler M., Elad Y., Baker R., 1984. Chlamydospore germination of Fusarium oxysporum f. sp. cucumerinum as affected by fluorescent and lytic bacteria from a Fusariumsuppressive soil. Phytopathology, 74, 1115-1124.

Sokal R. R., Rohlf J., 1969. Biometry, chap. 16, Ed. W. H. Freeman and Company.

Soussy C. J., Thibault M., Kitzis M. D., 1977. Activité antibactérienne comparée de 6 quinolones. Ann. Microbiol., 182B, 19-33.

Teintze M., Hossain M. B., Barnes C. L., Leong J., Van Der Helm D., 1981. Structure of ferric pseudobactin, a siderophore from plant growth promoting Pseudomonas. Biochemistry, 20, 6446-6457.

Xu G. W., Cross D. C., 1986. Field evaluations of the interactions among fluorescent pseudomonads, Erwinia carotovora and potato yields. Phytopathology, 76, 423-430. 